Research Article

\title{
Dynamical System Analysis of Thermal Convection in a Horizontal Layer of Nanofluids Heated from Below
}

\author{
J. M. Jawdat, ${ }^{1}$ I. Hashim, ${ }^{1}$ and S. Momani ${ }^{2}$ \\ ${ }^{1}$ School of Mathematical Sciences and Solar Energy Research Institute, \\ Universiti Kebangsaan Malaysia, 43600 Bangi, Selangor, Malaysia \\ ${ }^{2}$ Department of Mathematics, Faculty of Science, The University of Jordan, Amman 11942, Jordan
}

Correspondence should be addressed to I. Hashim, ishak_h@ukm.my

Received 10 May 2012; Accepted 26 July 2012

Academic Editor: Jun-Juh Yan

Copyright (C) 2012 J. M. Jawdat et al. This is an open access article distributed under the Creative Commons Attribution License, which permits unrestricted use, distribution, and reproduction in any medium, provided the original work is properly cited.

The effect of nanofluids on chaotic convection in a fluid layer heated from below was studied in this paper for low Prandtl number based on the theory of dynamical systems. A low-dimensional, Lorenz-like model was obtained using Galerkin-truncated approximations. The fourth-order Runge-Kutta method was employed to solve the nonlinear system. The results show that inhibition of chaotic convection can be observed when using nanofluids.

\section{Introduction}

Chaotic convection has attracted interest due to its wide applications in many natural systems, such as the time evolution of the magnetic field of celestial bodies, molecular vibrations, the dynamics of satellite in the solar system, the weather, ecology, and neurons. The transition from steady convection to chaos for low Prandtl number as studied by Vadasz and Olek [1] is sudden and occurs by a subcritical Hopf bifurcation producing a solitary limit cycle, which may be associated with a homoclinic explosion. This finding can be recovered from a truncated Galerkin expansion [2] which yields a system identical to the familiar Lorenz equations $[3,4]$. The work of Vadasz [5] suggests an explanation for the appearance of this solitary limit cycle via local analytical results. For the corresponding convection problem in a pure fluid, a similar approach was used by Vadasz $[6,7]$ to demonstrate similar results. Vadasz and Olek [8] showed that the route to chaos occurs by a period doubling sequence of bifurcations when the Prandtl number is moderate. Sheu [9] studied thermal convection in a fluid-saturated porous medium using a thermal nonequilibrium model to take account 
of the interphase heat transfer between the fluid and the solid. He found that the route to chaos was altered by interphase heat transfer and the nonequilibrium effect tends to stabilize steady convection. He also predicted an abrupt transition to chaos when interphase heat transfer is moderate and the porosity-modified conductivity ratio is small or moderate, while a period-doubling route to chaos was predicted with weak interphase heat transfer and a small-porosity-modified conductivity ratio. Jawdat abd Hashim [10] showed that the onset of chaotic convection in a porous medium for low Prandtl number can be enhanced by a uniform internal heat generation. The effects of a magnetic field on chaotic convection in porous media for low Prandtl number were investigated by Idris and Hashim [11]. They observed that the magnetic field could delay the convective motion in a saturated porous medium fluid layer. Mahmud and Hashim [12] studied the chaotic convection in a fluid layer heated from below when a constant, vertical magnetic field was applied. They showed that the chaotic convection can be suppressed or enhanced by the magnetic field.

Nanofluids, term proposed by Choi [13], are mixtures of base fluid such as water or ethylene-glycol with a very small amount of nanoparticles, having dimensions from 1 to $100 \mathrm{~nm}$ [14]. The onset of convection in a horizontal layer of a porous medium saturated by a nanofluid using linear instability theory, employing a model used for the nanofluid that incorporates the effects of Brownian motion and thermophoresis, was investigated analytically by Nield and Kuznetsov [15]. They showed that for a typical nanofluid (for which the Lewis number is large) the primary contribution of the nanoparticles was via a buoyancy effect coupled with the conservation of nanoparticles, with the contribution of nanoparticles to the thermal energy equation being a second-order effect. Alloui et al. [16] considered natural convection in a horizontal layer of a nanofluid with the horizontal boundaries heated and cooled by constant heat fluxes. They founded that the presence of nanoparticles in a fluid reduced the strength of flow field, this behaviour being more pronounced at low Rayleigh number. Also the temperatures on the solid boundaries were reduced by the presence of the nanoparticles when the strength of convection is high and enhanced when the strength of convection is low. The linear and nonlinear thermal instability in a rotating porous medium saturated by nanofluid, using Horton-Roger-Lapwood problem based on the Brinkman's Model, was studied by Bhadauria and Agarwal [14]. They observed that, for linear stability, the critical mode of onset of convection in most of the graphs was the oscillatory mode of convection. Also, they found that an exchange of stabilities from oscillatory convection to stationary convection holds as the value of wave number increased.

The aim of the present work is to study the influence of nanofluids on chaotic convection in a fluid layer heated from below extending the works of Vadasz [7] for low Prandtl number. The truncated Galerkin approximation was applied to the governing equations to deduce an autonomous system with three ordinary differential equations. This system was used to investigate the dynamic behaviour of thermal convection in the fluid layer and to elucidate the effects of nanofluids on the transition to chaos.

\section{Problem Formulation}

Consider an infinite horizontal fluid layer subject to gravity and heated from below with influence of nanofluids. A Cartesian coordinate system is used such that the vertical axis $z$ is collinear with gravity, that is, $\hat{e}_{g}=-\widehat{e}_{z}$.

The thermophysical properties of the nanofluids, considered in this study, given in Table 1, are assumed constant except for the density variation, which is determined based on the Boussinesq approximation and effected only for the gravity term in the momentum 
Table 1: Thermophysical properties of water and nanoparticles [16].

\begin{tabular}{lcccc}
\hline & $\rho\left(\mathrm{kg} \mathrm{m}^{-3}\right)$ & $C_{p}\left(\mathrm{Jkg}^{-1} \mathrm{~K}^{-1}\right)$ & $k\left(\mathrm{Wm}^{-1} \mathrm{~K}^{-1}\right)$ & $\beta \times 10^{5}\left(\mathrm{~K}^{-1}\right)$ \\
\hline $\mathrm{H}_{2} \mathrm{O}$ & 997.1 & 4179 & 0.613 & 21 \\
$\mathrm{Ag}$ & 10500 & 235 & 429 & 1.89 \\
$\mathrm{Cu}$ & 8933 & 385 & 401 & 1.167 \\
$\mathrm{Al}_{2} \mathrm{O}_{3}$ & 3970 & 765 & 40 & 0.85 \\
$\mathrm{TiO}_{2}$ & 4250 & 686.2 & 8.9538 & 0.9 \\
\hline
\end{tabular}

equation. Also, it is assumed that the base fluid and the nanoparticles are in thermal equilibrium and no slip occurs between them.

Subject to these conditions, the governing equations can be written as

$$
\begin{gathered}
\nabla \cdot V_{*}=0, \\
{\left[\frac{\partial V_{*}}{\partial t_{*}}+V_{*} \cdot \nabla V_{*}\right]=\frac{-1}{\rho_{n f}} \nabla p_{*}+v_{n f} \nabla^{2} V_{*}-\frac{(\rho \beta)_{n f}}{\rho_{n f}} \vec{g}\left(T_{*}-T_{c}\right),} \\
\frac{\partial T}{\partial t_{*}}+V_{*} \cdot \nabla T=\alpha_{n f} \nabla^{2} T
\end{gathered}
$$

where $V_{*}$ is the velocity, $T$ is temperature, and $p_{*}$ is pressure.

The effective density of the nanofluid, $\rho_{n f}$, is given as

$$
\rho_{n f}=(1-\phi) \rho_{f}+\phi \rho_{n p}
$$

and $\phi$ is the solid volume fraction of nanoparticles.

The thermal diffusivity of the nanofluid is

$$
\alpha_{n f}=\frac{k_{n f}}{\left(\rho C_{p}\right)_{n f}},
$$

where the heat capacitance of nanofluid is given by

$$
\left(\rho C_{p}\right)_{n f}=(1-\phi)\left(\rho C_{p}\right)_{f}+\phi\left(\rho C_{p}\right)_{n p} .
$$

The thermal expansion coefficient of nanofluid can be determined by

$$
(\rho \beta)_{n f}=(1-\phi)(\rho \beta)_{f}+\phi(\rho \beta)_{n p}
$$

The effective dynamic viscosity of the nanofluid is given by

$$
\mu_{n f}=\frac{\mu_{f}}{(1-\phi)^{2.5}}
$$


The thermal conductivity of the nanofluid can be determined by

$$
\frac{k_{n f}}{k_{f}}=\frac{k_{n p}+(n-1) k_{f}-(n-1) \phi\left(k_{f}-k_{n p}\right)}{k_{n p}+(n-1) k_{f}+\phi\left(k_{f}-k_{n p}\right)},
$$

where $n$ is an empirical shape factor for the nanoparticle. In particular, $n=3 / 2$ for cylindrical particles and $n=3$ for spherical ones (see [16]). In the present work, $n$ is set equal to 3 such that the results are restricted to spherical nanoparticles.

The following transformations will nondimensionalize (2.1)-(2.3)

$$
\begin{gathered}
V=\frac{H_{*}}{\alpha_{f}} V_{*}, \quad p=\frac{H_{*}^{2}}{\alpha_{f}^{2}} p_{*}, \quad \hat{t}=\frac{\alpha_{f}}{H_{*}^{2}} t_{*}, \quad T \Delta T_{c}=T_{*}-T_{c}, \\
x=\frac{x_{*}}{H_{*}}, \quad y=\frac{y_{*}}{H_{*}}, \quad z=\frac{z_{*}}{H_{*}},
\end{gathered}
$$

where $\widehat{t}$ is the time, $\left(T_{*}-T_{c}\right)$ is the temperature variations, and $\Delta T_{c}=\left(T_{H}-T_{c}\right)$ is the characteristic temperature difference.

The fluid layer with stress-free horizontal boundaries is considered. Hence, the solution must follow the impermeability conditions $V \cdot \widehat{e}_{n}=0$ and the stress-free condition $\partial u / \partial z=\partial v / \partial z=\partial^{2} w / \partial z^{2}=0$ on these boundaries, where $\widehat{e}_{n}$ is a unit vector normal to the boundary. The temperature boundary conditions are $T=1$ at $z=0$ and $T=0$ at $z=1$.

The governing equations can be represented in terms of a stream function defined by $u=-\partial \psi / \partial z$ and $w=\partial \psi / \partial x$, as for convective rolls having axes parallel to the shorter dimension (i.e., $y$ ) when $v=0$. Applying the curl $(\nabla \times)$ operator on (2.2) yields the following system of partial differential equations from (2.1)-(2.3):

$$
\begin{gathered}
{\left[\frac{1}{\operatorname{Pr}}\left(\frac{\partial}{\partial \widehat{t}}-\frac{\partial \psi}{\partial z} \frac{\partial}{\partial x}+\frac{\partial \psi}{\partial x} \frac{\partial}{\partial z}\right)-\bar{v} \nabla^{2}\right]\left(\nabla^{2} \psi\right)=\bar{\beta} \operatorname{Ra}\left(\frac{\partial T}{\partial x}\right)} \\
\frac{\partial T}{\partial \widehat{t}}-\frac{\partial \psi}{\partial z} \frac{\partial T}{\partial x}+\frac{\partial \psi}{\partial x} \frac{\partial T}{\partial z}=\bar{\alpha}\left(\frac{\partial^{2} T}{\partial x^{2}}+\frac{\partial^{2} T}{\partial z^{2}}\right)
\end{gathered}
$$

where

$$
\operatorname{Pr}=\frac{v_{f}}{\alpha_{f}}, \quad \operatorname{Ra}=\frac{\beta_{f} \Delta T_{c} g_{*} H_{*}^{3}}{\alpha_{f} v_{f}}
$$

are the Prandtl number, the Rayleigh number, respectively, and

$$
\bar{v}=\frac{v_{n f}}{v_{f}}, \quad \bar{\beta}=\frac{(\rho \beta)_{n f}}{\rho_{n f} \beta_{f}}, \quad \bar{\alpha}=\frac{\alpha_{n f}}{\alpha_{f}},
$$

and the boundary conditions for the stream function are $\psi=0$ on all solid boundaries. The values of $\bar{\nu}, \bar{\beta}$, and $\bar{\alpha}$ for nanoparticles are given in Table 2 . 
Table 2: Values of $\bar{\nu}, \bar{\beta}$, and $\bar{\alpha}$ for nanoparticles.

\begin{tabular}{lccc}
\hline & $\bar{v}$ & $\bar{\beta}$ & $\bar{\alpha}$ \\
\hline $\mathrm{Ag}$ & 0.770 & 0.675 & 1.181 \\
$\mathrm{Cu}$ & 0.813 & 0.697 & 1.167 \\
$\mathrm{Al}_{2} \mathrm{O}_{3}$ & 0.989 & 0.834 & 1.166 \\
$\mathrm{TiO}_{2}$ & 0.977 & 0.825 & 1.145 \\
\hline
\end{tabular}

The set of partial differential equations, (2.11), form a nonlinear coupled system and together with the corresponding boundary conditions will accept a basic motionless conduction solution.

\section{Reduced Set of Equations}

In order to obtain the solution to the nonlinear coupled system of partial differential equations in (2.11), we represent the stream function and temperature in the following form:

$$
\begin{gathered}
\psi=A_{11} \sin (\kappa x) \sin (\pi z), \\
T=1-z+B_{11} \cos (\kappa x) \sin (\pi z)+B_{02} \sin (2 \pi z) .
\end{gathered}
$$

This representation is equivalent to a Galerkin expansion of the solution in both the $x$ - and $z$-directions. Unlike in the works of Vadasz [7], we rescale the time and amplitudes with respect to their convective fixed points of the following form:

$$
\begin{aligned}
& X=\frac{\tilde{A}_{11}}{\sqrt{(\bar{\beta} \bar{\alpha} \lambda / \bar{v})(R-\bar{\alpha} \bar{v} / \bar{\beta})}}, \\
& Y=\frac{\widetilde{B}_{11}}{(\bar{v} / \bar{\beta}) \sqrt{(\bar{\beta} \bar{\alpha} \lambda / \bar{v})(R-\bar{\alpha} \bar{\nu} / \bar{\beta})}}, \\
& Z=\frac{-\widetilde{B}_{02}}{(R-\bar{\alpha} \bar{\nu} / \bar{\beta})} .
\end{aligned}
$$

We have the following system of ordinary differential equations:

$$
\begin{aligned}
& \dot{X}=\operatorname{Pr} \overline{\mathcal{v}}(Y-X) \\
& \dot{Y}=R\left(\frac{\bar{\beta}}{\overline{\bar{v}}}\right) X-\bar{\alpha} Y-\left(\frac{\bar{\beta}}{\bar{v}}\right)\left(R-\frac{\overline{\alpha \nu}}{\bar{\beta}}\right) X Z, \\
& \dot{Z}=\bar{\alpha} \lambda(X Y-Z)
\end{aligned}
$$


where

$$
R=\frac{\mathrm{Ra}}{\mathrm{Ra}_{c}}, \quad \operatorname{Ra}_{c}=\frac{\left(\kappa^{2}+\pi^{2}\right)^{3}}{\kappa^{2}}, \quad \lambda=\frac{8}{\left[\left(\kappa / \kappa_{\mathrm{cr}}\right)^{2}+2\right]},
$$

and the $\operatorname{dot}\left({ }^{(}\right)$denotes time derivatives $d() / d \tau$. When $\bar{v}=\bar{\beta}=\bar{\alpha}=1$, (i.e., $\phi=0$ ), system (3.3) reduces to the Vadasz system [7] ((2.11)-(2.12)). System (3.3) is equivalent to the Lorenz equations $[3,4]$, although with different coefficients. Using the wavenumber corresponding to the convection threshold, that is, $\kappa_{\mathrm{cr}}=\pi / \sqrt{2}$, in the definitions of $\lambda$ and $\operatorname{Ra}_{c}$ (3.4), yields $\lambda=8 / 3$ and $\mathrm{Ra}_{c}=27 \pi^{4} / 4$.

\section{Stability Analysis}

Stability analysis of the stationary solutions was performed in order to determine the nature of the dynamics about the fixed points. The nonlinear dynamics of a Lorenz-like system (3.3) has been analyzed and solved for $\operatorname{Pr}=10$ and $\lambda=8 / 3$. This rescaled system has three fixed points.

The first fixed point is $X_{1}=Y_{1}=Z_{1}=0$, corresponding to the motionless solution. The second and third fixed points corresponding to the convection solution are $X_{2,3}=Y_{2,3}=$ $\pm 1, Z_{2,3}=1$.

The stability of the first fixed point, $X_{1}=Y_{1}=Z_{1}=0$, is controlled by the zeros of the following characteristic polynomial equation for the eigenvalues $\sigma_{i}(i=1,2,3)$ :

$$
(-\bar{\alpha} \lambda-\sigma)\left[\sigma^{2}+(\bar{\alpha}+\operatorname{Pr} \bar{v}) \sigma+\operatorname{Pr}(\bar{v} \bar{\alpha}-R \bar{\beta})\right]=0 .
$$

The first eigenvalue, $\sigma_{1}=-\bar{\alpha} \lambda$, is negative since $\lambda=8 / 3$ and $\bar{\alpha}>0$. The other two eigenvalues are always real and given by

$$
\sigma_{2,3}=\frac{1}{2}\left[-(\bar{\alpha}+\operatorname{Pr} \overline{\mathcal{v}}) \pm \sqrt{(\bar{\alpha}+\operatorname{Pr} \overline{\mathcal{v}})^{2}+4 \operatorname{Pr}(R \bar{\beta}-\overline{\mathcal{v}} \bar{\alpha})}\right]
$$

$\sigma_{3}$ is also negative and $\sigma_{2}$ provides the stability condition for the motionless solution in the form $\sigma_{2}<0 \Leftrightarrow R<\bar{v} \bar{\alpha} / \bar{\beta}$. Therefore, the critical value of $R$, where the motionless solution loses stability and the convection solution (expressed by the other two fixed points) takes over, is obtained as

$$
R_{c 1}=R_{\mathrm{cr}}=\frac{\bar{v} \bar{\alpha}}{\bar{\beta}}
$$

which corresponds to $\operatorname{Ra}_{\mathrm{cr}}=\left(27 \pi^{4} / 4\right)(\bar{v} \bar{\alpha} / \bar{\beta})$. 
Table 3: Values of $R$ where $\sigma_{2}, \sigma_{3}$ become equal and complex conjugates and when the loss of stability occurred.

\begin{tabular}{lcc}
\hline & $\begin{array}{c}R\left(\sigma_{2}, \sigma_{3} \text { equal }\right) \\
\text { (complex conjugate) }\end{array}$ & $\begin{array}{c}R \\
\text { (loss of stability occurred) }\end{array}$ \\
\hline Vadasz & 1.35 & 24.737 \\
$\mathrm{Ag}$ & 1.825 & 37.503 \\
$\mathrm{Cu}$ & 1.845 & 36.305 \\
$\mathrm{Al}_{2} \mathrm{O}_{3}$ & 1.87 & 34.490 \\
$\mathrm{TiO}_{2}$ & 1.83 & 33.800 \\
\hline
\end{tabular}

The following cubic equation for the eigenvalues, $\sigma_{i}(i=1,2,3)$, controls the stability of the second and third fixed points of the rescaled system

$$
\sigma^{3}+(\bar{\alpha}+\bar{\alpha} \lambda+\operatorname{Pr} \bar{v}) \sigma^{2}+\left(\operatorname{Pr} \overline{\mathcal{v}} \bar{\alpha} \lambda+\frac{\bar{\alpha} \lambda \bar{\beta} R}{\bar{v}}\right) \sigma+2 \operatorname{Pr} \lambda\left(\bar{\alpha} \bar{\beta} R-\overline{\mathcal{v}} \bar{\alpha}^{2}\right)=0
$$

Equation (4.4) yields three eigenvalues, and the smallest eigenvalue $\sigma_{1}$ is always real and negative over the whole range of parameter values. The other two are real and negative at slightly supercritical values of $R$, such that the convection fixed points are stable, that is, simple nodes. These two roots move on the real axis towards the origin as the value of $R$ increases. For $\operatorname{Pr}=10, \lambda=8 / 3$, and $\phi=0.05$, these roots become equal when $R \cong 1.825$ for $\mathrm{Ag}, R \cong 1.845$ for $\mathrm{Cu}, R \cong 1.87$ for $\mathrm{Al}_{2} \mathrm{O}_{3}$, and $R \cong 1.83$ for $\mathrm{TiO}_{2}$ compared with $R \cong 1.35$ for Vadasz case. It is exactly at this point that these two roots become a complex conjugate. In any case, they still have negative real parts, and so the convection fixed points are stable, that is, spiral nodes. Both the imaginary and real parts of these two complex conjugate eigenvalues increase and extend over the imaginary axis as the value of $R$ increases. The real part becomes nonnegative at a value of $R$ given by

$$
R_{c 2}=\frac{\bar{v}^{2} \operatorname{Pr}(3 \bar{\alpha}+\bar{\alpha} \lambda+\bar{v} \operatorname{Pr})}{\bar{\beta}(\bar{v} \operatorname{Pr}-\bar{\alpha} \lambda-\bar{\alpha})} .
$$

Relation (4.5) is an extension of $R_{0}$ in [7] to the nanofluid case. At this point, the convection fixed points lose their stability and other (periodic or chaotic) solutions take over. The loss of stability of the convection fixed points for $\operatorname{Pr}=10, \lambda=8 / 3$, and $\phi=0.05$ using (4.5) is evaluated to be $R_{c 2}=37.503$ for $\mathrm{Ag}, R_{c 2}=36.305$ for $\mathrm{Cu}, R_{c 2}=34.490$ for $\mathrm{Al}_{2} \mathrm{O}_{3}$, and $R_{c 2}=33.800$ for $\mathrm{TiO}_{2}$ compared with $R_{0}=24.737$ for Vadasz loss of stability of the convection fixed points when $\phi=0$. For $\operatorname{Pr}=10, \lambda=8 / 3$, and $\phi=0.05$, the evolutions of the complex eigenvalues are presented in Figure 1. The value of $R$ where $\sigma_{2}, \sigma_{3}$ become equal and complex conjugate and when the loss of stability occurred are presented in Table 3.

\section{Results and Discussion}

In this section, some numerical simulations of the system (3.3) are presented for the time domain $0 \leq t \leq 210$. All calculations were done using MATLAB's built-in ODE45 based on the fourth-order Runge-Kutta method on double precision with stepsize 0.001 , fixing the 
Table 4: Comparison between Vadasz case $(\phi=0)$ and the nanofluid case for $\phi=0.05$ with $\operatorname{Pr}=10$, $\lambda=8 / 3$ for $R$ a solitary limit cycle signifying the loss of stability of the steady convection fixed points and the critical value of $R$ at which the chaotic behaviour solution occurs.

\begin{tabular}{lcc}
\hline & $R$ & $R$ \\
(limit cycle) & 24ritical value, chaotic behaviour) \\
\hline Vadasz & 23.474 & \\
$(\phi=0)$ & & 37.503 \\
$\mathrm{Ag}$ & 36.918 & 36.305 \\
$\mathrm{Cu}$ & 35.769 & 34.490 \\
$\mathrm{Al}_{2} \mathrm{O}_{3}$ & 34.032 & 33.800 \\
$\mathrm{TiO}_{2}$ & 33.352 & \\
\hline
\end{tabular}

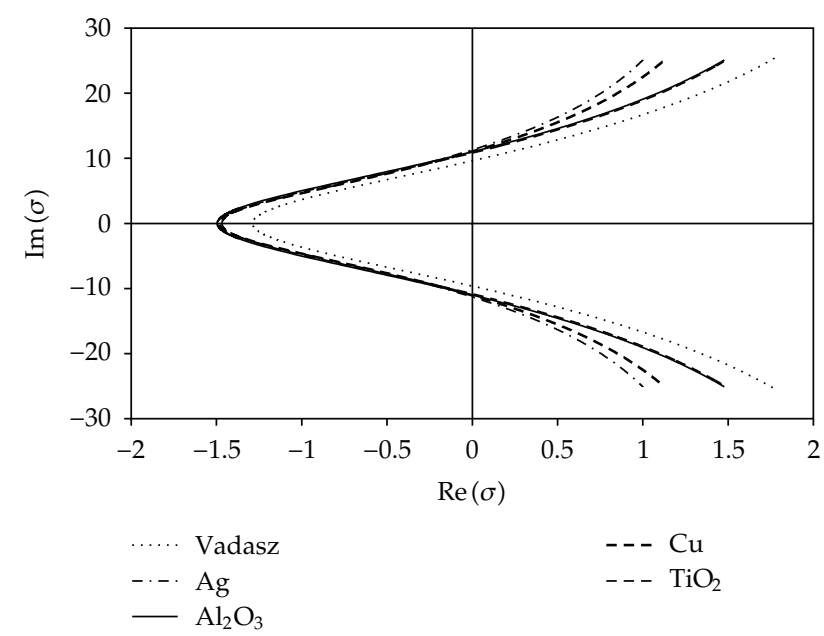

Figure 1: Evolution of the complex eigenvalues with increasing Rayleigh number, for $\operatorname{Pr}=10, \lambda=8 / 3$, and $\phi=0.05$.

values $\operatorname{Pr}=10, \lambda=8 / 3, \phi=0.05$, and taking the initial conditions $X(0)=Y(0)=0.8$ and $Z(0)=0.92195$.

Comparing to Vadasz case [7], the critical value of $R$ in each case is greater than the critical value in Vadasz case. Thus the onset of chaotic convection is delayed. A comparison between Vadasz case and the case under study is mentioned in Table 4 for $R$ being a solitary limit cycle signifying the loss of stability of the steady convection fixed points and the critical value of $R$ at which the chaotic behaviour solution occurs.

In the case of $\mathrm{Ag}$, we found that at $R_{c 1}=1.346$ obtained from (4.3), the motionless solution loses stability and the convection solution take over. Also the values of the eigenvalues $\sigma_{2}$ and $\sigma_{3}$ from (4.4) become equal and complex conjugate when $R \cong 1.825$. At the value of $R=36.918$, we obtain a solitary limit cycle signifying the loss of stability of the steady convection fixed points. When $R=37.503$, the convection fixed points lose their stability and a chaotic solution takes over. The evolution of trajectories over time in the state space for two values of Rayleigh number ( $R$ where the solution is limit cycle and the critical value of $R$ ) is presented in Figure 2(a).

In the case of $\mathrm{Cu}$, we found that at $R_{c 1}=1.361$ obtained from (4.3), the motionless solution loses stability and the convection solution takes over. Also the values of the 
$(\mathrm{Ag}, R=36.918)$

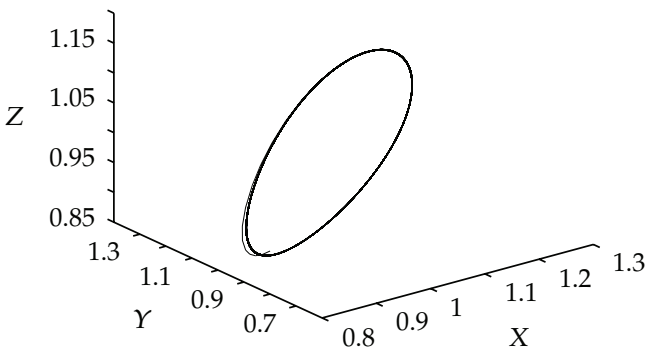

$(\mathrm{Ag}, R=37.50319652)$

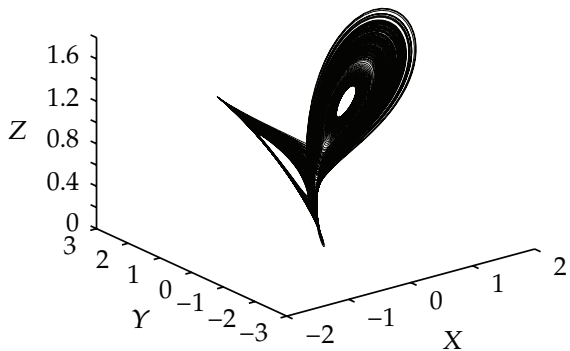

(a) $\mathrm{Ag}$

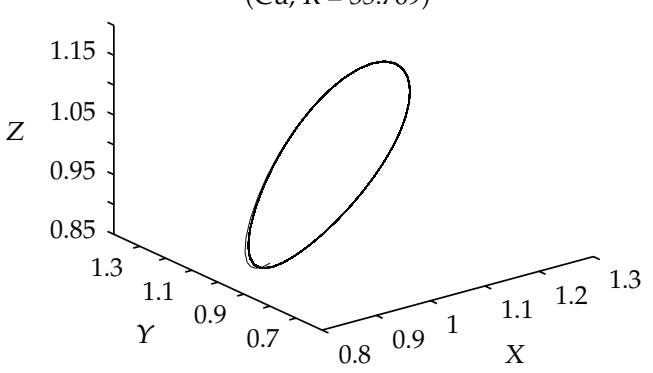

$(\mathrm{Cu}, R=36.30496243)$

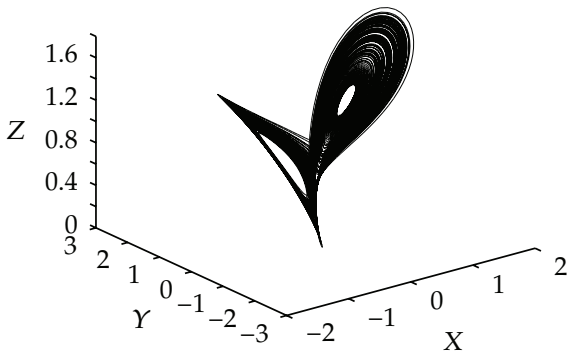

(b) $\mathrm{Cu}$

$\left(\mathrm{Al}_{2} \mathrm{O}_{3}, R=34.0316\right)$
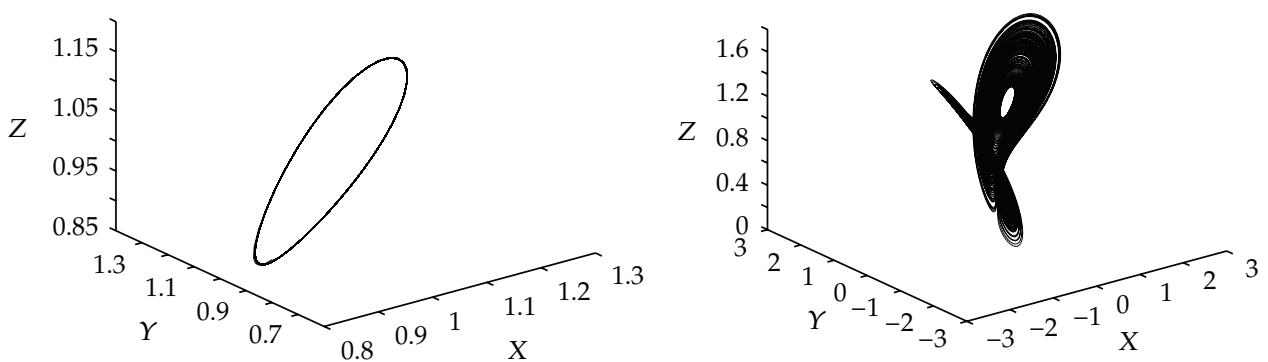

(c) $\mathrm{Al}_{2} \mathrm{O}_{3}$

$\left(\mathrm{TiO}_{2}, R=33.3517\right)$

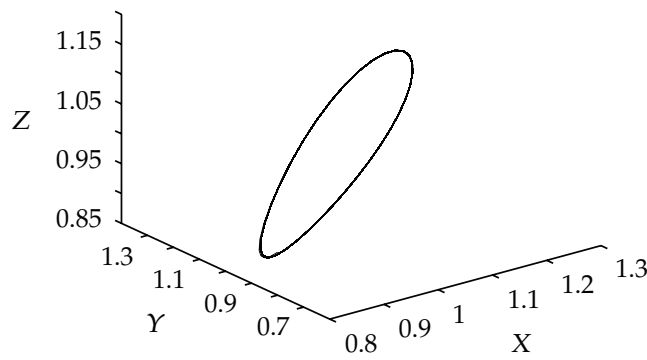

$\left(\mathrm{TiO}_{2}, R=33.80018476\right)$

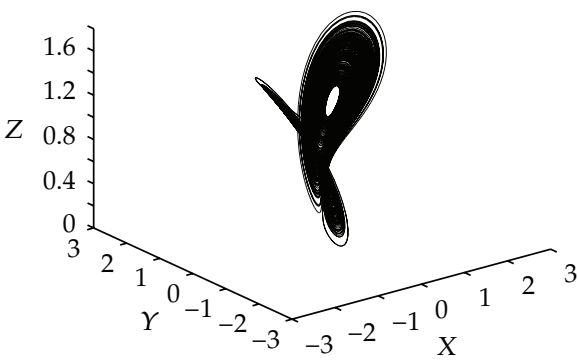

(d) $\mathrm{TiO}_{2}$

Figure 2: Computational results for the evolution of trajectories over time in the state space for two values of Rayleigh number ( $R$ where the solution is limit cycle and the critical value of $R$ ). The graphs represent the projection of the solution data points onto $X-Y-Z$ plane for $\operatorname{Pr}=10, \lambda=8 / 3$, and $\phi=0.05$. 


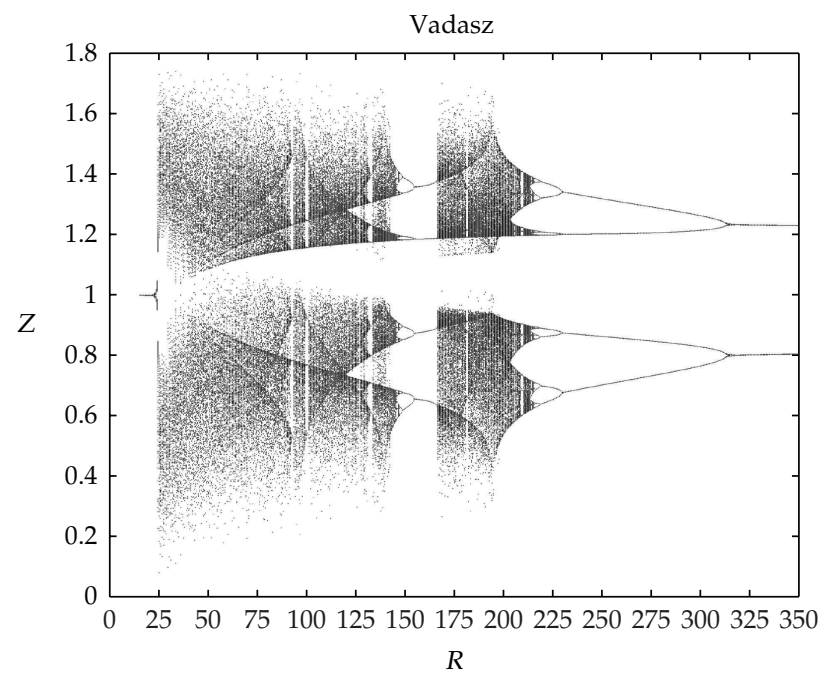

(a)

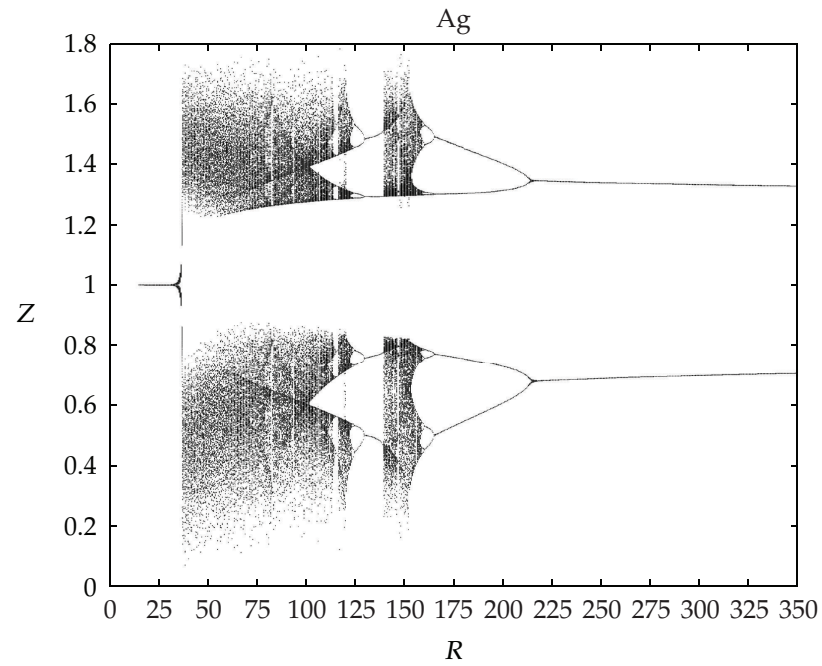

(b)

Figure 3: Bifurcation diagrams of $Z$ versus $R$ representing maxima and minima of the posttransient solution of $Z(t)$ with $\operatorname{Pr}=10$ and $\lambda=8 / 3$ for Vadasz case and Ag case with $\phi=0.05$.

eigenvalues $\sigma_{2}$ and $\sigma_{3}$ from (4.4) become equal and complex conjugate when $R \cong 1.845$. When $R=35.769$, we obtain a solitary limit cycle signifying the loss of stability of the steady convection fixed points. At the value of $R=36.305$, the convection fixed points lose their stability and a chaotic solution takes over. The evolution of trajectories over time in the state space for two values of Rayleigh number ( $R$ where the solution is limit cycle and the critical value of $R$ ) is presented in Figure 2(b).

In the case of $\mathrm{Al}_{2} \mathrm{O}_{3}$, we found that at $R_{c 1}=1.384$ obtained from (4.3), the motionless solution loses stability, and the convection solution takes over. Also the values of the eigenvalues $\sigma_{2}$ and $\sigma_{3}$ from (4.4) become equal and complex conjugate when $R \cong 1$.87. We obtain a solitary limit cycle signifying the loss of stability of the steady convection fixed points 
$(\mathrm{Ag}, R=24.737)$

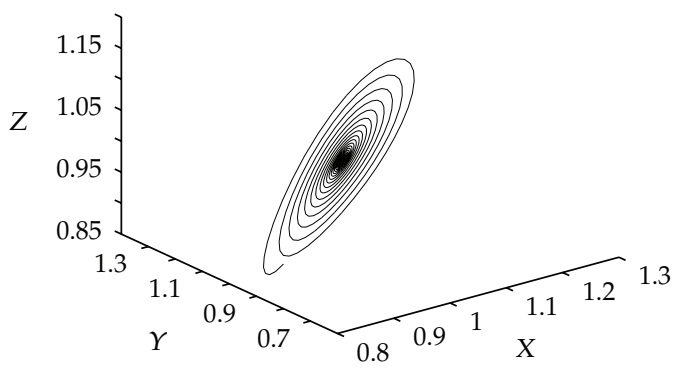

(a)

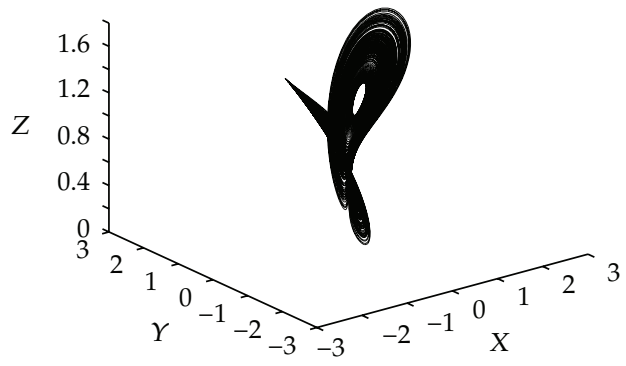

$(\mathrm{Ag}, R=150)$
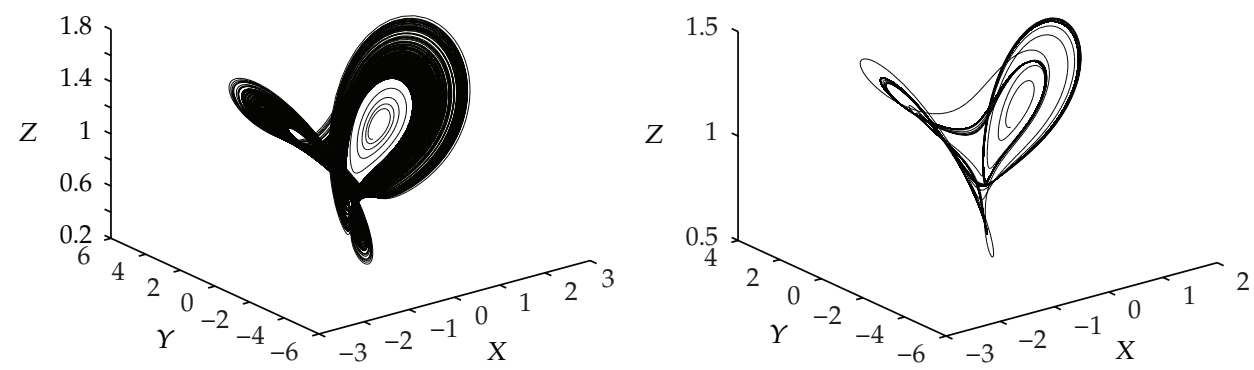

(b)

$(\mathrm{Ag}, R=175)$
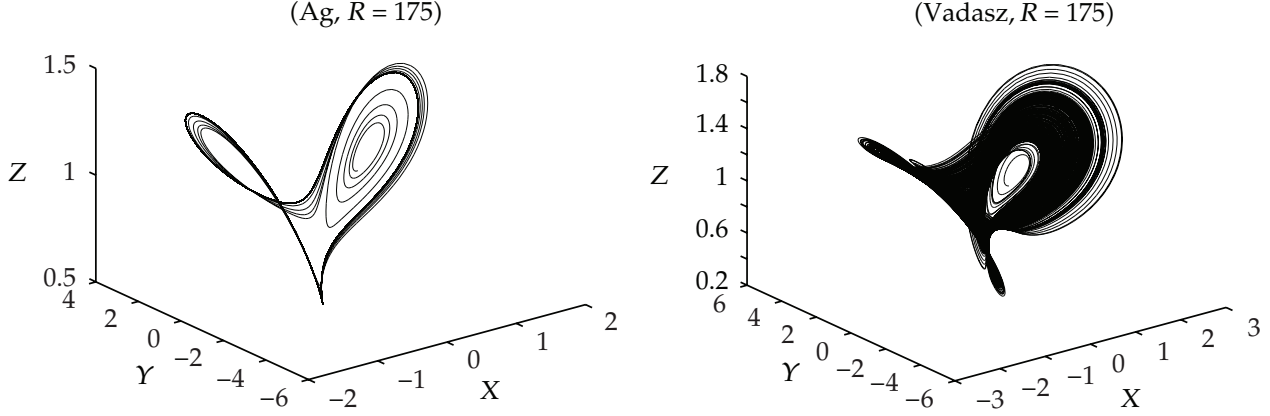

(c)

$(\mathrm{Ag}, R=250)$
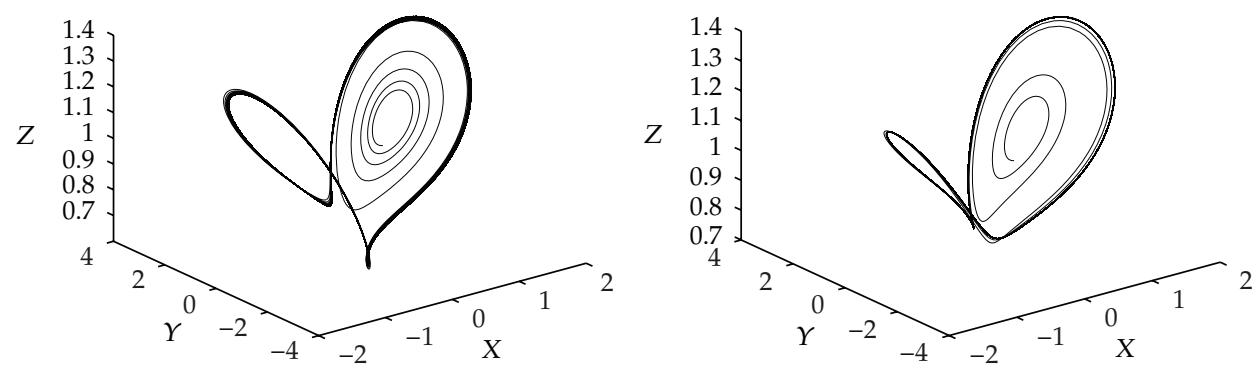

(d)

Figure 4: Computational results for the evolution of trajectories over time in the state space for different values of Rayleigh number $R$. The graphs represent the projection of the solution data points onto $X-Y-Z$ plane when $\operatorname{Pr}=10$ and $\lambda=8 / 3$ for Vadasz case and Ag case with $\phi=0.05$. 
at the value of $R=34.032$. When $R=34.490$, the convection fixed points lose their stability and a chaotic solution takes over. The evolution of trajectories over time in the state space for two values of Rayleigh number ( $R$ where the solution is limit cycle and the critical value of $R$ ) is presented in Figure 2(c).

In the case of $\mathrm{TiO}_{2}$, we found that at $R_{c 1}=1.358$ obtained from (4.3), the motionless solution loses stability and the convection solution takes over. Also the values of the eigenvalues $\sigma_{2}$ and $\sigma_{3}$ from (4.4) become equal and complex conjugate when $R \cong 1.83$. At the value of $R=33.352$, we obtain a solitary limit cycle signifying the loss of stability of the steady convection fixed points. We can observe that the convection fixed points lose their stability and a chaotic solution takes over at $R=33.800$. The evolution of trajectories over time in the state space for two values of Rayleigh number ( $R$ where the solution is limit cycle and the critical value of $R$ ) is presented in Figure 2(d).

From Figure 2, we observe that $\mathrm{Ag}$ and $\mathrm{Cu}$ nanoparticles have similar chaotic behaviour, while $\mathrm{Al}_{2} \mathrm{O}_{3}$ and $\mathrm{TiO}_{2}$ have similar chaotic behaviour also but with different shape. Thus the oxide- or dioxide-free nanoparticles have different chaotic behaviour than oxide or dioxide one.

For a close look, we choose Ag nanoparticles and present the bifurcation diagrams, in Figure 3, in terms of maxima and minima in the posttransient values of $Z$ versus $R$ for Vadasz case and Ag case with $15 \leq R \leq 350$ and a resolution of $\Delta R=0.5$.

From Figure 3, we observe that the chaotic behaviour is delayed with decreasing the chaotic region when using the Ag nanoparticles. Also, we see the difference in the behaviour for them. For $R=24.737$, the critical value in Vadasz case, the trajectories approach the fixed point on a spiral behaviour for Ag, while it be chaotic one in Vadasz case as in Figure 4(a). In addition, when $R=150$, it is periodic in Vadasz case compared with chaotic for Ag (Figure 4(b)). The converse behaviour is true for $R=175$ as shown in Figure 4(c). Finally, although the behaviour is periodic for both cases when $R=250$ as in Figure 4(d), it appears in different shapes.

\section{Conclusions}

In this paper, we have studied chaotic behaviour in a fluid layer subject to gravity and heated from below under the effect of nanofluids for low Prandtl number. Comparing with Vadasz case, we notice that the onset of chaotic convection can be delayed under the influence of nanofluids. This means that the stability region can be increased using nanofluids. As a conclusion, the transition from steady convection to chaos depends on the properties of the nanoparticles.

\section{References}

[1] P. Vadasz and S. Olek, "Weak turbulence and chaos for low Prandtl number gravity driven convection in porous media," Transport in Porous Media, vol. 37, no. 1, pp. 69-91, 1999.

[2] P. Vadasz and S. Olek, "Transitions and chaos for free convection in a rotating porous layer," International Journal of Heat and Mass Transfer, vol. 41, no. 11, pp. 1417-1435, 1998.

[3] E. N. Lorenz, "Deterministic non-periodic flow," Journal of the Atmospheric Sciences, vol. 20, pp. 130142, 1963.

[4] C. Sparrow, The Lorenz Equations: Bifurcations, Chaos, and Strange Attractors, vol. 41 of Applied Mathematical Sciences, Springer, New York, NY, USA, 1982.

[5] P. Vadasz, "Local and global transitions to chaos and hysteresis in a porous layer heated from below," Transport in Porous Media, vol. 37, no. 2, pp. 213-245, 1999. 
[6] P. Vadasz, "On the homoclinic orbit for convection in a fluid layer heated from below," International Journal of Heat and Mass Transfer, vol. 42, no. 19, pp. 3557-3561, 1999.

[7] P. Vadasz, "Subcritical transitions to chaos and hysteresis in a fluid layer heated from below," International Journal of Heat and Mass Transfer, vol. 43, no. 5, pp. 705-724, 2000.

[8] P. Vadasz and S. Olek, "Route to chaos for moderate Prandtl number convection in a porous layer heated from below," Transport in Porous Media, vol. 41, no. 2, pp. 211-239, 2000.

[9] L. J. Sheu, "An autonomous system for chaotic convection in a porous medium using a thermal nonequilibrium model," Chaos, Solitons and Fractals, vol. 30, no. 3, pp. 672-689, 2006.

[10] J. M. Jawdat and I. Hashim, "Low Prandtl number chaotic convection in porous media with uniform internal heat generation," International Communications in Heat and Mass Transfer, vol. 37, no. 6, pp. 629-636, 2010.

[11] R. Idris and I. Hashim, "Effects of a magnetic field on chaos for low Prandtl number convection in porous media," Nonlinear Dynamics, vol. 62, no. 4, pp. 905-917, 2010.

[12] M. N. Mahmud and I. Hashim, "Effects of a magnetic field on chaotic convection in fluid layer heated from below," International Communications in Heat and Mass Transfer, vol. 38, no. 4, pp. 481-486, 2011.

[13] S. U. S. Choi, "Enhancing thermal conductivity of fluids with nanoparticles," in Proceedings of the ASME Fluids Engineering Division, vol. 231, pp. 99-105, November 1995.

[14] B. S. Bhadauria and S. Agarwal, "Natural convection in a nanofluid saturated rotating porous layer: a nonlinear study," Transport in Porous Media, vol. 87, no. 2, pp. 585-602, 2011.

[15] D. A. Nield and A. V. Kuznetsov, "Thermal instability in a porous medium layer saturated by a nanofluid," International Journal of Heat and Mass Transfer, vol. 52, no. 25-26, pp. 5796-5801, 2009.

[16] Z. Alloui, P. Vasseur, and M. Reggio, "Natural convection of nanofluids in a shallow cavity heated from below," International Journal of Thermal Sciences, vol. 50, no. 3, pp. 385-393, 2011. 


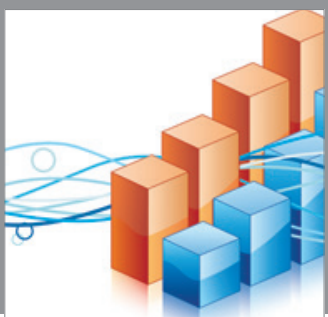

Advances in

Operations Research

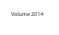

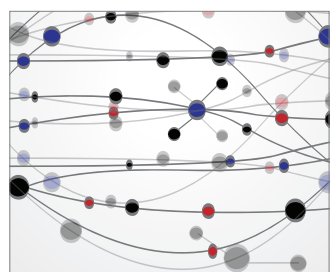

\section{The Scientific} World Journal
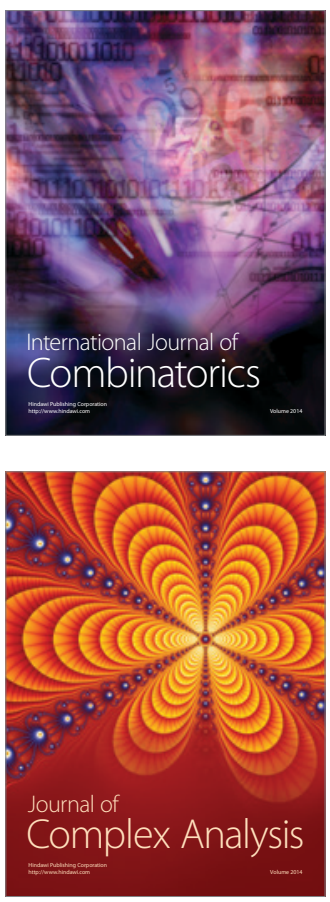

International Journal of

Mathematics and

Mathematical

Sciences
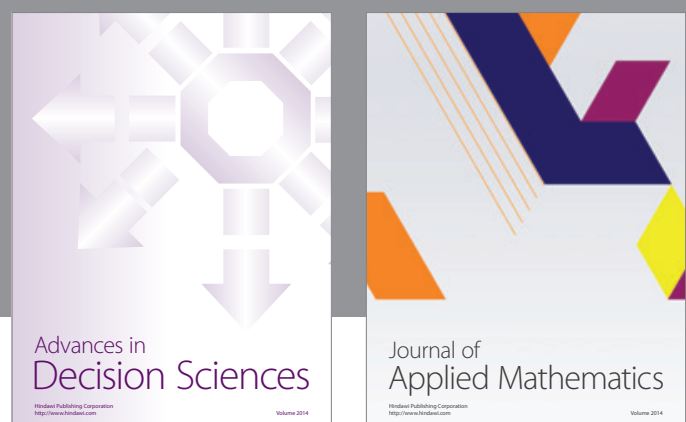

Journal of

Applied Mathematics
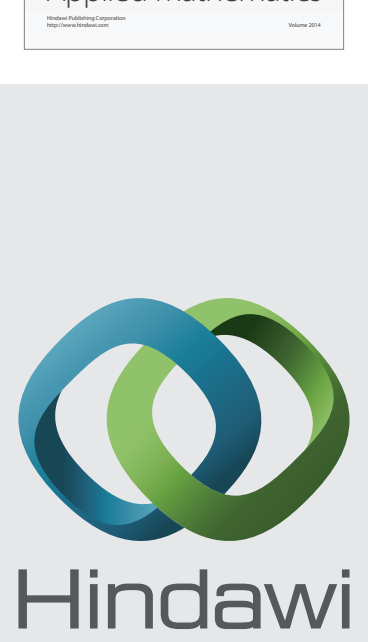

Submit your manuscripts at http://www.hindawi.com
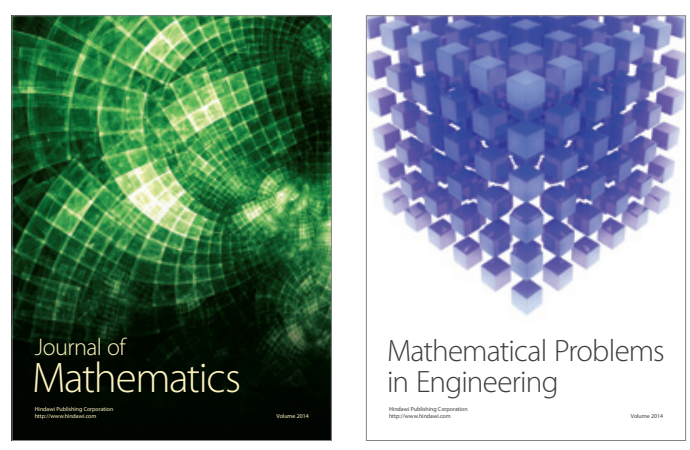

Mathematical Problems in Engineering
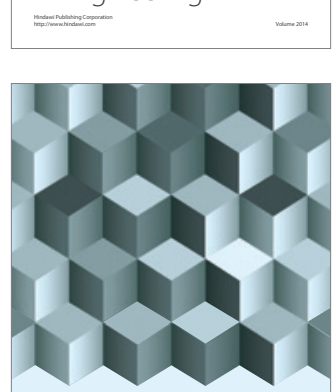

Journal of

Function Spaces
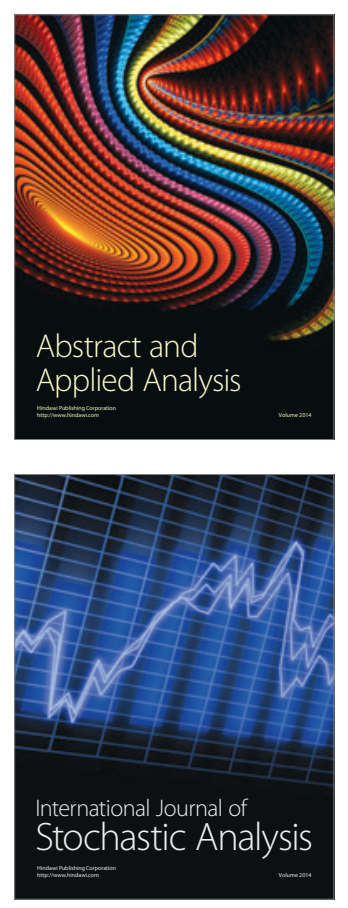

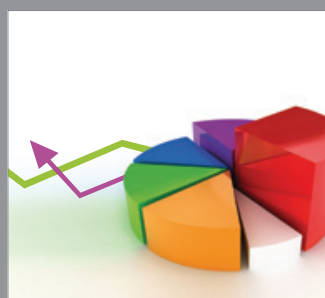

ournal of

Probability and Statistics

Promensencen
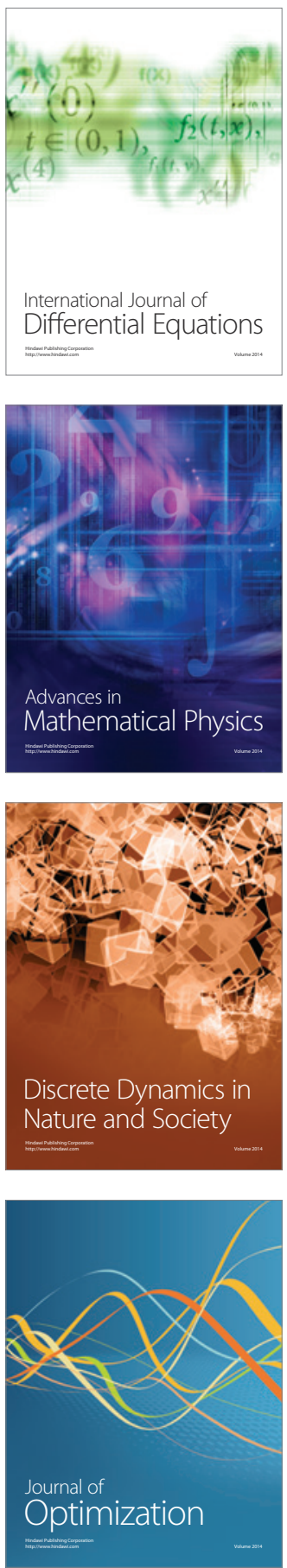